\title{
Diacronie
}

Studi di Storia Contemporanea

$N^{\circ} 9,1 \mid 2012$

Quando la classe operaia andava in paradiso

\section{Due vie democratiche al socialismo?}

PCI, PCF e il concetto di nazione tra il 1944 e il 1947

\section{Martina Martignoni}

\section{(2) OpenEdition \\ Journals}

\section{Edizione digitale}

URL: http://journals.openedition.org/diacronie/2911

DOI: 10.4000/diacronie.2911

ISSN: 2038-0925

Editore

Association culturelle Diacronie

Notizia bibliografica digitale

Martina Martignoni, «Due vie democratiche al socialismo? », Diacronie [Online], № 9, 1 | 2012,

documento 1, Messo online il 29 janvier 2012, consultato il 10 mai 2019. URL : http://

journals.openedition.org/diacronie/2911; DOI : 10.4000/diacronie.2911 


\title{
Diacronie
}

\section{Due vie democratiche al socialismo? PCI, PCF e il concetto di nazione tra il 1944 e il 1947}

\author{
Martina MARTIGNONI*
}

Partito Comunista Italiano e Partito Comunista Francese, i due maggiori partiti comunisti d'Europa, hanno una storia che sembra scorrere parallela dalla fine della Seconda Guerra Mondiale fino al 1956. Attraverso l'analisi della costruzione di un discorso attorno al tema della nazione, tra il 1944 e il 1947, vediamo effettivamente quali sono state le (tante) somiglianze e le (poche ma significative) differenze che hanno segnato la storia dei comunisti italiani e francesi.

\section{Introduzione}

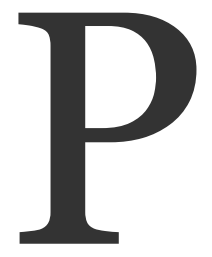

artito Comunista Italiano (PCI) e Partito Comunista Francese (PCF), storicamente i maggiori partiti comunisti europei, sono stati spesso messi a confronto. Tra le interpretazioni storiche più recenti, sicuramente quella di Marc Lazar ${ }^{1}$ è la più interessante perché tenta di ridimensionare la differenza tra i due partiti, differenza in passato invece fortemente sottolineata e oggi ancora presente nel pensare comune degli ex-comunisti italiani: un PCF fortemente stalinista a fronte di un PCI più autonomo rispetto alle scelte sovietiche, un PCF più operaio e un PCI più aperto a differenti componenti sociali, un PCF più radicale e un PCI più disponibile al dialogo con le altre forze politiche.

\footnotetext{
${ }^{1}$ LAZAR, Marc, Maisons Rouges: Les Partis communistes français et italien de la Libération à nos jours, Paris, Aubier, 1992.
} 
Marc Lazar nel suo Maisons Rouges sostiene appunto che nella storiografia che ha posto a confronto PCI e PCF si rintracciano due elementi da mettere in discussione: una "opposizione primordiale" tra i due partiti e una valutazione insidiosa che consiste nel presentare il PCI sotto i suoi aspetti migliori in rapporto al $\mathrm{PCF}^{2}$. Alla storiografia (soprattutto italiana) che sottolinea l'originalità del PCI, Lazar contrappone uno studio che tende a relativizzare questa originalità, e allo stesso tempo a minimizzare la supposta autonomia del PCI rispetto all'URSS. La relativizzazione dell'originalità del PCI riguarda soprattutto gli anni del dopoguerra, per poi riconoscere le differenze, che, soprattutto dal 1956, segneranno una divergenza più netta delle strade intraprese dai due partiti.

Di seguito proverò a mostrare come in quest'analisi sfuggano alcuni elementi che secondo me segnano invece una differenza tra i due partiti. Differenza che non vuole suggerire che ci siano alcuni aspetti migliori nell'uno o nell'altro, ma semplicemente riconoscere diverse composizioni, strategie e tattiche, elementi importanti per capire anche le difformità politiche tra Francia e Italia, nel passato come nel presente.

Prenderò qui in considerazione gli anni che vanno dalla fine della Seconda Guerra Mondiale all'uscita nel 1947 dei due partiti comunisti dai governi di unità nazionale. Il focus dell'analisi ruota attorno alla costruzione di un discorso pubblico sul tema della nazione da parte dei comunisti.

I richiami alla nazione e al dovere nazionale, al popolo come soggetto della trasformazione sociale, riecheggiano in modo simile, ma non identico, nelle parole dei comunisti francesi e italiani. Come i comunisti riuscirono a conciliare questo registro con il discorso di classe? Fu il VII Congresso dell'Internazionale del 1935 che dette, a tutti i partiti comunisti, il compito di concentrarsi sul territorio nazionale come spazio d'intervento politico con l'obiettivo di abbattere il fascismo. Sia il PCF sia il PCI accolsero e fecero proprie queste decisioni, operando un cambiamento nella loro politica che si tradusse in una trasformazione, anche netta, del loro discorso pubblico.

Analizzerò qui la costruzione di una retorica, di un linguaggio, di un discorso pubblico appunto (che poi ovviamente si traducono in decisioni politiche che ebbero un peso) da parte del PCI e del PCF attorno al tema della nazione.

Gli elementi all'interno di questa retorica che metterò a fuoco sono: l'inserimento della storia dei partiti comunisti nella storia nazionale; la costruzione di una politica nazionale; le nazionalizzazioni e l'incremento della produttività; la Costituzione.

${ }^{2}$ LAZAR, Marc, op. cit., p. 17. 


\section{I comunisti nella storia della nazione}

PCI e PCF, partecipando alla lotta di liberazione dal nazi-fascismo, s’inserirono a pieno diritto nella storia nazionale di Italia e Francia. Consapevoli della forza acquisita durante la Resistenza rispetto alle altre parti politiche, sfruttarono al massimo la situazione per rafforzare la loro posizione: uno degli strumenti fu l'inserirsi nella storia della nazione, non semplicemente quella di cui furono attori, ma quella passata, rintracciandovi radici, antenati, insegnamenti.

Uno dei motivi che li spinse a quest'operazione fu sicuramente la necessità di difendersi dagli attacchi degli altri partiti che li accusavano (soprattutto in Francia) di non essere formazioni realmente interessate al proprio paese, ma all'URSS.

Se il riferimento del PCF alla storia rivoluzionaria della Francia, dal 1789 al 1871, appare un'operazione non così complessa, più difficoltà vi furono per il PCI nell'inserirsi in una storia di unità nazionale realizzata da una casa reale e a seguito di invasioni territoriali, più che da movimenti popolari. Il PCI, infatti, fino al 1935 rifiutò piuttosto nettamente (distinguendosi in questo da Giustizia e Libertà) una lettura progressista dell'unità d'Italia e la denominazione della Resistenza al fascismo come un "secondo Risorgimento". Così si esprimeva Togliatti nel 1931:

I borghesi tengono curvi i contadini sotto il giogo del capitale. Le fantasie sul "secondo Risorgimento" sono fatte solo per nascondere questa realtà. 3

Secondo Togliatti (nel 1931) il Risorgimento è stato un movimento stentato, al quale le masse non hanno partecipato, che ha mancato di un carattere rivoluzionario perché la borghesia italiana non ha voluto porre fine alla feudalità e al problema della terra per paura che il suo potere fosse travolto. Stando così le cose, non esisteva, secondo il segretario del partito, nessun Risorgimento da riprendere o da proseguire da parte dell'antifascismo democratico. Le questioni, infatti, che non furono risolte nel Risorgimento dovevano essere risolte contro di esso e contro la classe che ne era stata protagonista, la borghesia. Ma un cambiamento piuttosto netto si registrò dopo il 1935, anno del VII Congresso dell'Internazionale Comunista, in cui i partiti decisero di occupare il campo dello scontro nazionale. Da quel momento in poi, e soprattutto dopo la vittoria della Resistenza, i riferimenti al Risorgimento ci furono (in particolare attraverso la figura di Garibaldi, che dette il nome alle stesse brigate partigiane

3 ERCOLI [TOGLIATTI, Palmiro], "Sul movimento di "Giustizia e Libertà"», in Lo Stato Operaio, V, 1931, pp. 463-473. 
comuniste) e furono utilizzati per cercare di legare la storia della classe operaia a quella nazionale (e dunque, di conseguenza, quella del PCI alla nazione).

«Noi siamo il partito della classe operaia e non rinneghiamo e non rinnegheremo mai, questa nostra qualità. Ma la classe operaia non è stata mai estranea agli interessi della nazione. [...] Operai e artigiani furono il nerbo dei combattenti delle Cinque giornate di Milano. Furono gli operai, insieme coi migliori rappresentanti dell'intellettualità, l'anima della resistenza degli ultimi baluardi della libertà italiana nell'anno successivo. Operai e artigiani troviamo nelle legioni di Garibaldi; li troviamo dappertutto dove ci si batte e si muore per la libertà e per l'indipendenza del paese».4

Quanto al Partito comunista francese, il suo inserimento nella storia nazionale si mosse su due assi, a partire da due fatti fondamentali della storia francese (e non solo) moderna: la Rivoluzione del 1789 e la Comune del 1871. Mentre dal riferimento alla prima, il PCF trasse soprattutto l'esempio storico della sua "teoria dell'ascesa delle classi” e il legame storico tra Rivoluzione francese e Rivoluzione d'ottobre, dalla seconda trasse il discorso del "popolo contrapposto ai traditori della Francia" e l'insegnamento storico che il popolo ha bisogno della guida del partito comunista per poter vincere.

Come la borghesia fu la classe ascendente che sostituì l'ormai morente aristocrazia alla guida del paese attraverso la Rivoluzione, così nel dopoguerra la classe operaia, nuova classe ascendente, doveva sostituire la classe borghese rivelatasi incapace di guidare il paese. Il riferimento più usato per sostenere quest'assioma fu il famoso discorso di Sieyès Che cos'è il Terzo Stato? pronunciato nel gennaio 1789:

Au temps de Sieyès, c'est la bourgeoisie révolutionnaire qui était portée par les conditions historiques à la tête de ce Tiers État qui se confondait avec la nation. Aujourd'hui, en raison de l'évolution sociale et économique, c'est la classe ouvrière qui a pris la tête du Tiers État moderne: elle anime la nation et elle seule peut l'émanciper des privilégiées modernes qui, comme ceux de 1789, sont une charge pour la nation, mais ne sauraient faire partie. 5

L'accostamento, inoltre, tra giacobini e bolscevichi e l'idea di una continuazione della Rivoluzione francese in quella bolscevica furono alimentati dal pensiero

4 TOGLIATTI, Palmiro, «La politica di unità nazionale dei comunisti», in ID., Opere, vol. 5, 1944-1955, Roma, Editori Riuniti, 1984, p. 14.

5 COGNIOT, George, «Classe ouvrière et nation», in Cahiers du communisme, 12, octobre 1945. 
socialista $^{6}$. Si è voluto vedere nella Rivoluzione d'ottobre un compimento delle istanze liberatrici aperte ma non portate fino in fondo dalla Rivoluzione francese; quest'ultima quindi, in una lettura del genere, perde la sua specificità per diventare un "eventomadre" di un altro, ormai nuovo punto di riferimento nella storia del progresso ${ }^{7}$.

È evidente quindi che il riferimento alla Rivoluzione francese fatto dal PCF portò in qualche modo quello che potremmo definire un doppio vantaggio: da una parte inserì il partito nella storia nazionale, dall'altra alimentò questa narrazione che legava Francia e URSS. Spesso i due paesi furono messi a paragone dal PCF e si affermò che come la Francia fu fonte d'ispirazione e di speranza per i democratici e i rivoluzionari d'Europa e non solo, così l'Unione Sovietica fu il paese a cui guardavano coloro che credevano e che desideravano un mondo più giusto, più democratico. Come Robespierre e SaintJust, figli della Francia, incarnarono un momento di progresso umano, così aveva fatto Lenin nella prima metà del Novecento ${ }^{8}$. Ancora una volta si proseguì nel tentativo di smorzare gli atteggiamenti apertamente anticomunisti e antisovietici dimostrati dagli avversari politici del PCF.

La seconda narrazione costruita dal PCF in cui il riferimento storico fu usato in funzione presente fu quella legata alla storia della Comune del 1871. Questo argomento venne utilizzato quando dovevano difendere la necessità di ricostruire su basi popolari la Costituzione e la rinascita francesi dopo la guerra. La "paura del popolo", spesso agitata dal PCF, sarebbe stata la causa della nascita del governo di Vichy, e poteva essere la causa di una futura disgrazia per la Francia. La Comune, dunque, come fonte d’insegnamento perché certi errori (la mancanza di fiducia nel popolo, il tradimento del paese) non si ripetessero.

Encore plus qu'en 1870 la peur du peuple a été l'élément essentiel de la trahison des trusts et de leurs valets qui, dés 1933 avaient mise sur Hitler. En 1940, comme en 1870-1871, Paris et le peuple étaient l'ennemi $\mathrm{n}^{\circ}{ }_{1}$ pour les gens du gouvernement: la peur du peuple conduisait une fois de plus à la trahison de la France. Pourquoi faut-il donc que nous soyons encore obligés de rappeler aujourd'hui que sans le peuple rien n'est possible?9

\footnotetext{
6 KONDRATIEVA, Tamara, «Les "bolcheviks-jacobins": révélation d'une analogie», Communisme, 20-21, 1989, pp. 71-87. 7 FURET, François, Critica della Rivoluzione francese, Roma-Bari, Laterza, 1995.

8 CASSAGNES, Jean, «Le $21^{\circ}$ anniversaire de la mort de Lénine», in Cahiers du Communisme, 3, janvier 1945 .

9 l'Humanité, 28 mai 1945.
} 
La Commune reste donc extraordinairement vivant par ses leçons. Elle nous a appris où résidaient les bases de la trahison des intérêts nationaux et les conditions de l'indépendance du pays. Elle a indiqué que l'autorité d'un gouvernement, à l'intérieur comme à l'extérieur, reposait sur sa liaison avec les couches profondes du peuple et sa volonté de traduire les initiatives créatrices de ce dernier. Si la prochaine Constituante entend établir en France une démocratie inébranlable qui évite au pays le retour des malheurs récents, elle se doit de tenir compte des formes politiques et sociales de la Commune de Paris, tout en les adaptant aux circonstances nouvelles différentes. ${ }^{10}$

\section{Una politica (comunista) nazionale}

«La seule politique authentiquement française», così il segretario del PCF Maurice Thorez definì la pratica politica del suo partito nel novembre del 1945, e in quell'occasione ricordò che:

alors que la classe ouvrière est aujourd'hui l'héritière de tout ce qui a fait la France, la détentrice des vertus authentiques du peuple français, c'est elle qui défend sans cesse le pur intérêt de la nation française. Le Parti communiste [...] est le parti le plus profondément national du pays [...].11

Era la classe operaia, nella lettura del PCF, la classe che difendeva l'interesse della nazione francese. Perché la classe operaia avrebbe dovuto difendere gli interessi della nazione e non i propri? Perché avrebbe dovuto essere più interessata alle sorti della nazione francese piuttosto che, ad esempio, alle sorti del movimento operaio internazionale? Jacques Duclos, membro del comitato centrale del PCF e numero due del partito, sosteneva che «la classe ouvrière ne peut pas concevoir sa libération sans la libération de la Nation» ${ }^{12}$.

Con "liberazione della Nazione" i comunisti intendevano la liberazione dai traditori e dai trusts, oltre che dagli invasori.

Dal momento in cui la vittoria del nazismo nel 1933 in Germania aveva segnato un cambiamento nella situazione internazionale, per $i$ comunisti francesi l'internazionalismo aveva fatto il suo tempo e la questione che da allora si poneva non

1o BERLIOZ, Joanny, «La Commune de Paris», in Cahiers du Communisme, 5, mars 1945.

11 l'Humanité, 6 novembre 1945.

12 l'Humanité, 28 ottobre 1944. 
era più democrazia borghese o socialismo, ma democrazia o fascismo ${ }^{13}$. Non solo cambiarono i termini del conflitto, ma anche lo spazio di questo, e allora fu la nazione che si pose come la "nuova tappa della lotta di classe". Ci fu uno sforzo continuo da parte dei dirigenti del PCF nel rendere chiari i motivi di questa politica alla base, probabilmente perché così chiari non erano. Cercarono appoggio nei maestri del "socialismo scientifico" per spiegare i motivi che avvicinavano le sorti della classe operaia alle sorti della nazione. I riferimenti furono principalmente al testo di Stalin $I l$ marxismo e la questione nazionale (pubblicato in Francia nel 1939 e in Italia nel 1948), al Lenin che si batté per l'emancipazione del suo paese dallo zarismo, a Engels e Marx che sostennero i popoli in lotta per la loro indipendenza (irlandesi, polacchi, ungheresi, francesi nel 1870), al Manifesto del Partito comunista, la cui celebre frase «gli operai non hanno patria» venne interpretata differentemente dal solito: il PCF sostenne infatti che non si trattava di un'affermazione "positiva" ma di un "rimprovero" e di una "condanna”, di un'esortazione affinché gli operai conquistassero una patria ${ }^{14}$.

La controparte principale della politica nazionale del PCF furono i trusts, le grandi compagnie che sostennero il nazismo e il governo di Vichy, definite dal PCF "traditrici della nazione".

La rinascita, affermavano i comunisti, poteva avvenire solo sottraendo ai trust il controllo della produzione. La guida di questo processo doveva stare nelle mani della classe operaia, alla testa delle nuove forze nazionali. Il compito della classe operaia e del PCF era di riunire tutte le masse popolari di tutti gli orizzonti politici, organizzarle e indirizzarle sulla via del redressement francese. Questa era la nuova tappa della lotta di classe: «la lutte de la Nation contre les trusts sous la direction de la classe ouvrière»15. Se i trust, in quanto traditori della patria, dividevano il paese, la classe operaia doveva essere invece in grado di unirlo; e per far questo prima di tutto doveva essere unita al suo interno. L'operazione politica auspicata dal PCF fu la creazione di un Parti Ouvrier Français (un tentativo di unire Partito comunista e Partito socialista che non andò in porto).

Anche il PCI si mobilitò per spiegare alla base i cambiamenti della politica comunista. Nel rapporto ai quadri dell'organizzazione comunista napoletana dell'11

13 RUFFE, Hubert, «Sur la dissolution de l'Intérnationale communiste», in Cahiers du communisme, 6, april 1945 .

14 MONMOUSSEAU, Gaston, «Une nouvelle étape de la lutte des classes», in Cahiers du communisme, 8-9, giugno-luglio 1945; CASSAGNES, Jean, "Unité ouvrière et unité nationale», in Cahiers du communisme, 8-9 giugno-luglio 1945; COGNIOT, Georges, «Classe ouvrière et nation", in Cahiers du Communisme, 12, ottobre 1945; "Bibliographie: Staline: le marxisme et la question nationale», in Cahiers du communisme, 10, ottobre 1946.

15 MONMOUSSEAU, Gaston, op. cit. 
aprile 1944 Togliatti spiegò alla sua base quale doveva essere la nuova politica dei comunisti. Se nel passato, prima della Seconda Guerra Mondiale, il partito comunista si era limitato a denunciare e criticare la politica della classe dirigente, nell'Italia ancora in gran parte occupata dai tedeschi doveva essere in grado di indicare una via di uscita e di guidare il popolo verso di essa. Quello che chiedeva il segretario del PCI era che i comunisti fossero un'avanguardia capace di pensare agli interessi della nazione. Prendere in mano la questione nazionale significava prima di tutto portare a termine per il PCI, nell'unità di tutte le forze antifasciste, la liberazione del paese, ma significava anche strappare il discorso sulla nazione all'ideologia fascista e dargli un nuovo contenuto.

La bandiera degli interessi nazionali, che il fascismo ha trascinato nel fango e tradito, noi la raccogliamo e la facciamo nostra; liquidando per sempre la ideologia da criminali del fascismo e i suoi piani funesti di brigantaggio imperialista, tagliando tutte le radici della tirannide mussoliniana noi daremo alla vita della nazione un contenuto nuovo, che corrisponda ai bisogni, agli interessi, alle aspirazioni delle masse del popolo. ${ }^{16}$

I continui appelli del PCI all'unità delle forze antifasciste devono essere letti principalmente alla luce della paura che il fascismo potesse, sotto altre forme rispetto a quelle del ventennio, tornare. Se consideriamo che fino al 2 giugno del 1946 con il referendum istituzionale, quindi a più di un anno di distanza dalla fine della guerra, non si poteva ancora sapere nemmeno se l'Italia sarebbe stata una monarchia o una repubblica, possiamo comprendere come le preoccupazioni del PCI per il futuro istituzionale dell'Italia avessero un senso. Mentre, infatti, in Francia si era già posto a referendum un progetto di costituzione, in Italia non si era ancora eletta un'assemblea costituente. Questo ci può far capire la differente piega che prende il discorso nazionale dei due partiti comunisti in Italia e in Francia. Il discorso del PCI fu molto incanalato verso il sostegno alla forma repubblicana dello stato come unica forma democratica possibile, e sugli sforzi necessari per arrivare alla sua realizzazione, sforzi necessariamente da compiere al fianco di tutte le forze antimonarchiche e antifasciste: socialisti, cattolici, repubblicani.

Con la guerra di liberazione, diceva sostanzialmente il PCI, lotta di classe, antifascismo e patriottismo venivano a coincidere; in questa situazione si costituì un'alleanza tra operai, contadini, piccola borghesia e una parte della stessa borghesia

16 TOGLIATTI, Palmiro, «La politica di unità nazionale dei comunisti», cit., p. 14. 
monopolistica per l'indipendenza nazionale, contro il fascismo, per la democrazia. E in questo blocco la classe operaia sarebbe dovuta diventare la forza d'avanguardia di una larga alleanza.

La politica nazionale del Partito comunista riflette questa realtà; essa pone il problema nazionale al centro della politica della classe operaia i cui interessi si identificano con quelli della grande maggioranza del popolo italiano e quindi con gli interessi nazionali. Essa è la sola giusta applicazione del marxismo-leninismo nelle nuove condizioni storiche createsi al movimento operaio. ${ }^{17}$

La lettura sulla necessità storica che la classe operaia si interessasse della questione nazionale fu dunque, anche se con diverse sfumature di analisi, condivisa da PCI e PCF: l'avvento del fascismo aveva spostato la politica del partito comunista dall'antitesi democrazia borghese-dittatura del proletariato, all'antitesi fascismo-democrazia; le "caste capitalistiche reazionarie" avevano abbandonato e tradito le posizioni nazionali che nel passato gruppi dirigenti della borghesia progressiva avevano tenuto; la classe operaia aveva combattuto per la difesa della nazione e della libertà e si trovava a capo delle forze di difesa degli "interessi della nazione".

\section{Nazionalizzazioni e aumento della produzione}

Sia in Francia sia in Italia durante la Resistenza sorsero esperienze di autogestione delle fabbriche da parte operaia. In Francia si trattò di un centinaio di casi nel sud del paese, tra Marseille, Toulouse, Montluçon, Berliet e Lyon. In Italia i Consigli di Gestione sorsero durante l'insurrezione del nord per difendere le fabbriche abbandonate dai nazisti e furono adottati dal Comitato di Liberazione Nazionale Alta Italia il 25 aprile 1945. Sia in Francia sia in Italia, però, sembra che i comunisti non dettero importanza e appoggio a queste esperienze, mostrandosi invece favorevoli alle nazionalizzazioni.

Se per lungo tempo il PCF aveva sposato le tesi guesdiste contrarie alle nazionalizzazioni (e favorevoli invece alle socializzazioni ${ }^{18}$ ), come aveva dimostrato durante tutte le discussioni riguardo al programma del Front Populaire degli anni '3o,

17 Ibidem.

18 Altra cosa rispetto alla nazionalizzazione era la socializzazione, la quale costituiva una trasgressione del quadro capitalista ed era gestita dai lavoratori organizzati che esercitano il potere, quindi da una classe e non dalla nazione tutta. 
nel secondo dopoguerra invece la linea del PCF le sostenne. Per i comunisti, infatti, prima della Seconda Guerra Mondiale, lo Stato era visto come un nemico e finché la classe operaia non se ne fosse impadronita, le nazionalizzazioni sarebbero potute essere solo statalizzazioni, quindi un'operazione gestita dalla borghesia. In questo i comunisti differivano dai socialisti che intendevano positivamente la nazionalizzazione in quanto forma di appropriazione collettiva da parte della nazione nella quale forze produttrici e consumatori potevano intervenire nella gestione dell'impresa nazionalizzata allo stesso titolo dello Stato. Per i comunisti, invece, c'era corrispondenza tra Stato e Nazione, che non erano intesi come corpi separati. Il PCF del secondo dopoguerra virò però su un appoggio alle nazionalizzazioni, che escludeva dunque un sostegno alle socializzazioni (ovvero l'esperienza di autogestione delle fabbriche). C'è dunque in questo passaggio una nuova interpretazione delle nazionalizzazioni e dell'idea stessa di nazione. Sembra che i comunisti si fossero convinti del fatto che esistesse una corrispondenza tra classe operaia, popolo e nazione, grazie alla quale le nazionalizzazioni sarebbero state gestite dal popolo, e dunque dal proletariato. Dare appoggio alle socializzazioni avrebbe significato forse non riuscire a costruire un fronte largo di sostegno alle nazionalizzazioni, che incontrarono, invece, il consenso di quasi tutte le forze politiche, compreso De Gaulle. Ciò che è certo è che nella situazione del secondo dopoguerra le nazionalizzazioni rappresentarono per i comunisti francesi una misura politica prima ancora che economica: furono lo strumento attraverso il quale si poterono combattere i trust, i grandi monopoli, i capitalisti. Le nazionalizzazioni per il PCF furono quindi strettamente legate all'epurazione ${ }^{19}$.

Il PCI, più che trattare le esperienze di autogestione come esperienze di classe, le esaltò come dimostrazione dell'interesse degli operai verso la Nazione, come atto eroico di salvataggio della produzione, delimitando il compito dei lavoratori a un monitoraggio della produzione, in modo che essa fosse fatta «negli interessi della nazione» e addirittura arrivando a sostituire «interesse di classe» con «gretto interesse corporativo», in opposizione a «interessi e ideali che si identificano con quelli della Nazione tutta ${ }^{20}$. Legata alle nazionalizzazioni fu la questione della produzione e delle politiche economiche. Secondo il PCF era necessario nazionalizzare non solo gran parte della produzione (e in primo luogo le miniere di carbone) ma anche banche e assicurazioni (operazione che trovò il consenso di gran parte delle forze politiche e andò in porto). Nazionalizzazione significava da una parte sfiducia politica dei

19 ANDRIEU, Claire, LE VAN, Lucette, PROST, Antoine, Les nationalisations de la libération. De l'utopie au compromis, Paris, Presses de la Fondation National de Sciences Politiques, 1987 ${ }^{20}$ SERENI, Emilio, «I consigli di gestione», in Rinascita, II, 9-10, settembre-ottobre 1945. 
monopoli e dall'altra dimostrazione della maggiore produttività del popolo. Questo nella pratica diventò ciò che dallo stesso PCF fu denominata la "bataille de la production" (già durante il governo del Front Populaire e sotto il governo di Vichy, in forme e per motivi diversi, ci si era appellati alla produttività dei lavoratori e si era lanciata una "battaglia del carbone"21). Dalla fine della guerra e fino al maggio 1945 la produzione di carbone fu per i minatori francesi un obiettivo che s'inseriva e portava a compimento l'azione della Resistenza. Durante questi mesi i lavoratori, sulla spinta del partito comunista e della CGT, aumentarono la produzione da $674 \mathrm{~kg}$ nel settembre 1944 a 910 kg nel marzo 1945. In primavera però i minatori, stanchi, sotto alimentati e scontenti di un'epurazione giudicata insufficiente, si rifiutarono di fornire uno sforzo supplementare. Scioperi scoppiarono lungo la primavera, in più bacini, malgrado l'opposizione della CGT, e il rendimento scese di nuovo al di sotto dei $900 \mathrm{~kg}$. A questo fatto il PCF rispose lanciando appunto la battaglia della produzione, alla cui costruzione collaborarono rappresentanti della CGT e della Fédération du sous-sol, e la direzione stessa delle Houillères Nationales Nord-Pas-de-Calais (già nazionalizzate)22. Il Partito comunista dovette convincere i lavoratori, in particolare i minatori, di una "verità" non così evidente, ovvero che il loro interesse si confondeva con quello di tutti. I temi utilizzati nella costruzione di un discorso che potesse arrivare ai minatori furono principalmente l'interesse nazionale e il patriottismo. A questi temi, usati generalmente da tutte le forze politiche che auspicavano un aumento della produzione, se ne aggiunsero alcuni della propaganda comunista e della CGT: l'appello alla sensibilità dei minatori, ai valori tradizionali della corporazione e alla sua storia.

Importanti in questo senso furono tre discorsi pronunciati da Thorez tra il luglio 1945 e l'agosto 1946, nei quali il segretario si scagliò contro gli scioperi e l'assenteismo, in particolare quello giovanile. Affermò (in tono paternalistico) che era necessario superare la crisi della moralità che stava vivendo la Francia, in particolare tra i giovani, e dichiarò che gli scansafatiche non sarebbero mai stati dei buoni comunisti.

Gli argomenti su cui si cercò di fare leva furono l'appartenenza alla categoria e l'orgoglio che si doveva provare ad essere in primo luogo minatori, e in secondo luogo comunisti. Per onorare al meglio queste due appartenenze era necessario fare uno sforzo di produzione, ripetere l'atto d'eroismo dimostrato durante la Liberazione, fare ciò che il partito chiedeva.

${ }^{21}$ TREMPÉ, Rolande, Les trois batailles du charbon (1936-1947), Paris, Editions la Découverte, 1989.

22 SERENI, Emilio, «I consigli di gestione», cit. 
Il PCF puntò da una parte sul patriottismo e dall'altra sull'appartenenza di classe, facendo convergere, attraverso il senso del dovere, interesse nazionale e interesse di classe: «Produire, faire du charbon, c'est la forme la plus élevée de votre devoir de classe, de votre devoir de Français! ${ }^{23}$.

Anche il PCI sviluppò un discorso per sostenere la "solidarietà nazionale", che si articolava assieme a quello sulla funzione nazionale della classe operaia e a quello della sulla ricostruzione costituendo una sorta d'ideologia, come la definisce Camillo Daneo ${ }^{24}$. "Solidarietà nazionale" era un discorso che tentava di imporre ai più ricchi una morale che li obbligasse a sentirsi coinvolti nelle sorti della nazione e del popolo e che li portasse dunque a fare una politica che in qualche modo giovasse a tutti e non solo ad alcuni. Ma il rovescio della medaglia della solidarietà nazionale significava senso morale di appartenenza alla nazione da parte della classe operaia, che si traduceva in: impegno nell'aumentare la produttività, collaborazione con il capitale attraverso i consigli di gestione, «difesa attiva dei ceti medi produttori».

Anche per il PCI l'aumento della produzione era un compito fondamentale della classe operaia. Intervenendo al convegno economico dell'agosto 1945 Togliatti elencò quali erano le prime cose da fare nell'ambito economico, e il primo punto era quello dell'aumento della produzione:

La prima cosa da farsi è, secondo me, l'appello agli operai perché, dappertutto dove essi lavorano, aumentino il rendimento del lavoro. D'ora in avanti questo deve essere uno dei punti fondamentali della nostra agitazione. [...] La classe operaia deve sapere che l'aumento della produttività del lavoro è una delle condizioni per riuscire a creare in Italia un regime democratico[...]. Naturalmente, l'appello all'aumento dell'operosità nell'interesse di tutti dev'essere accompagnato dall'impegno del governo a fare una politica di solidarietà nazionale e a combattere la speculazione e il sabotaggio. ${ }^{25}$

Rispondendo sulle pagine di Rinascita alle critiche di un delegato che osservava che non può esserci solidarietà laddove c'è contrasto d'interessi e antagonismo di classi, Scoccimarro spiegò che il partito invocava la solidarietà nazionale per individuare e rendere visibili al popolo quali erano le forze che operavano contro gli interessi nazionali, e pose tutto il discorso sul piano della moralità:

23 l'Humanité, 19 febbraio 1945.

24 DANEO, Camillo, La politica economica della ricostruzione: 1945-1949, Torino, Einaudi, 1975.

${ }_{25}$ TOGLIATTI, Palmiro, I problemi economici, in ID., Opere, vol. 5, 1944-1955, cit., p. 169. 
Solidarietà nazionale vuol dire richiamare le classi abbienti al dovere morale di venire incontro ai bisogni elementari delle classi che nulla possiedono e che sono state gettate dalla guerra e dall'invasione nella più squallida miseria. [...] Ma il nostro appello alla solidarietà nazionale ha un significato ancor più vasto: è il costume e la concezione di vita della classe operaia, che noi opponiamo al costume e alla concezione della borghesia. [...] quando noi oggi poniamo il problema della funzione nazionale della classe operaia, cioè della sua funzione politica dirigente nella vita del Paese, poniamo contemporaneamente il problema che tutto ciò che essa porta in sé di positivo in tutti i campi: economico, politico e morale deve divenire patrimonio di tutta la nazione, deve dare la propria impronta alla vita nazionale. ${ }^{26}$

\section{Costituzione}

Ciò che mi sembra dia l'idea delle profonde diversità tra PCI e PCF è la diversa attitudine mostrata nel "cristallizzare" il potere conquistato, nell'istituzionalizzare la propria idea politica, nel contribuire dunque alla stesura della Costituzione. Ambedue i partiti erano consapevoli dell'impossibilità di raggiungere il socialismo a breve ed elaborarono due concetti simili ("democrazia progressiva" e "democrazia popolare") che dettero l'idea di una dilazione nel tempo (e nelle modalità non rivoluzionarie) della conquista di esso. All'interno di questo cammino, per entrambi la difesa della democrazia fu un passaggio imprescindibile per «rendere il socialismo più vicino nell'avvenire» ${ }^{27}$. All'interno di questo passaggio però, l'uso che PCI e PCF fecero del principale istituto democratico, il parlamento, mi sembra significativamente differente. Un primo punto di differenziazione sta nell'idea stessa di Costituzione. Questa, pensavano i comunisti francesi, deve essere la consacrazione legislativa di ciò che è già stato conquistato, e non un'enunciazione di principi eterni e universali, non un programma in cui si espongono gli scopi dell'avvenire.

26 SCOCCIMARRO, Mauro, «Una discussione sulla nostra politica», in Rinascita, II, 12, dicembre 1945 .

${ }^{27}$ FAJON, Etiènne, «Un projet de constitution démocratique de la République française», Cahiers du Communisme, 13, dicembre 1945. 
L'objet essentiel de la Constitution, c'est de fixer la forme de l'Etat dans un pays donné, à une époque donnée, en fonction des rapports économiques, sociaux et politiques qui existent dans ce pays à cette époque. ${ }^{28}$

Com'è noto i comunisti italiani pensarono invece ad una costituzione che ponesse le basi di alcuni principi fondamentali, seppur non raggiungibili a breve termine, che avrebbero guidato a lungo il paese (e ancora oggi chi sostiene l'intoccabilità della costituzione italiana si riferisce a questo principio). Nella sua forma generale, la Costituzione italiana, sia per com'è stata pensata dal PCI, sia per come effettivamente è stata redatta, è una costituzione che dichiara principi e propositi assai lontani dalla realtà materiale dell'Italia di quegli anni; è una Costituzione che guarda al futuro, dichiara principi che dovranno guidare per molto tempo il paese (idea che s'inseriva pienamente nel progetto di "democrazia progressiva"). Interessante in questo senso è il dialogo che Piero Calamandrei e Togliatti ebbero nell'Assemblea plenaria della Commissione per la Costituzione proprio sulla natura di alcuni articoli della prima parte della Costituzione italiana ${ }^{29}$.

Calamandrei propose di inserire delle norme che dichiaravano alcuni diritti individuali dei cittadini (diritti sociali come quello al lavoro, alla casa, all'assistenza contro invalidità e vecchiaia ecc.) in un preambolo e non tra le norme giuridiche, constatando che di fatto questi diritti non erano garantiti e che non sarebbe stato possibile nell'immediato garantirli, e che più che l'enunciazione di diritti affermati erano invece «affermazioni generiche, desideri, speranze politiche». Calamandrei riteneva (sulla scorta dell'esempio della costituzione bolscevica) che affinché questi fossero diritti in senso giuridico sarebbe stato necessario precisare chi era obbligato e quali erano i mezzi pratici per rendere effettivi questi diritti. A una critica di tipo giuridico Togliatti rispose con argomentazioni politiche. Il segretario del PCI affermò che in Italia e in Europa, dati i rapporti di classe determinatisi, si potesse arrivare a una profonda trasformazione senza una rivoluzione com'era avvenuto in Russia. La Costituzione doveva quindi tenere conto di questo:

[...] se sancisse soltanto quello che esiste oggi in Italia, non corrisponderebbe a quello che la grande maggioranza del popolo desidera dalla Costituzione. La nostra Costituzione deve dire qualche cosa di più, deve avere un carattere programmatico,

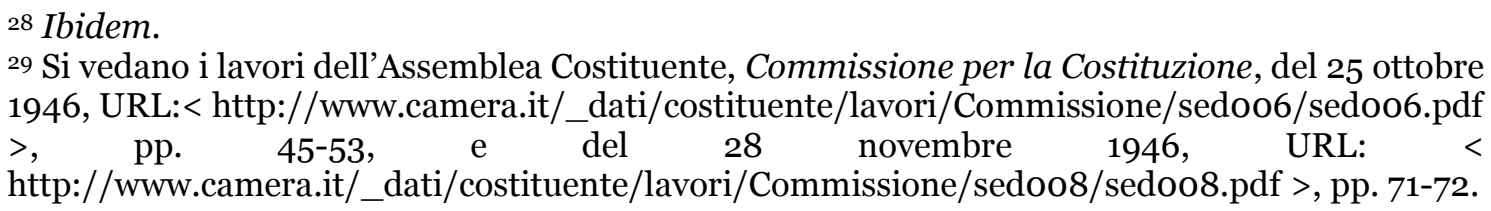


almeno in alcune delle sue parti, e particolarmente in quelle parti in cui si afferma la necessità di dare un nuovo contenuto ai diritti dei cittadini, un contenuto sociale [...]. Ritiene perciò che i diritti sociali debbano essere affermati in concreto in articoli della Costituzione, i quali avranno un carattere normativo, ma in pari tempo anche un carattere programmatico. Si tratta di un avviamento, di un impegno, di un orientamento alla creazione di un nuovo ordinamento sociale e quindi di una nuova legalità. $3^{0}$

Viene da chiedersi se il sancire costituzionalmente come diritti dei diritti nella realtà non affermati, non significhi in qualche modo svuotarli di significato, e farli diventare costituzionalmente "utopie" che nessuno ha l'obbligo (né giuridico, né politico) di mettere in pratica.

Altro punto di differenziazione è la battaglia sull'Assemblea unica. Sia il PCI che il PCF concordavano sul fatto che fosse necessario che i nuovi parlamenti fossero costituiti da un'Assemblea unica eletta a suffragio universale, garanzia per una reale sovranità popolare. Mentre il PCF, appoggiato dalla SFIO, ma avversato fortemente da tutte le altre forze politiche, portò avanti con forza questa idea e riuscì ad inserirla nel primo progetto di costituzione approvato dall'assemblea costituente, il PCI si mostrò debole nel difendere l'assemblea unica e ben presto accantonò l'idea. Sicuramente il diverso rapporto di forza di cui godevano i due partiti all'interno delle assemblee giocò un ruolo (il PCF insieme ai socialisti formava una maggioranza schiacciante, mentre il PCI non aveva il pieno appoggio dei socialisti italiani), ma comunque il generale atteggiamento del PCI fu difensivo e molto più disposto alla concertazione con DC e PSI.

Se consideriamo insieme il progetto di costituzione del PCF che prevedeva un'assemblea unica e sovrana e quello della creazione del Parti ouvrier, che sarebbe dovuto nascere dalla fusione di PCF e Parti Socialiste, non possiamo non leggere in queste scelte il tentativo del Partito comunista francese di conquistare il potere attraverso il parlamento, cioè attraverso la conquista da parte del Parti Ouvrier della maggioranza dell'Assemblea nazionale.

Da parte del PCI, invece, il compromesso fu teso a trasformare lo Stato in senso democratico, a costruire una Costituzione che garantisse tutte le libertà democratiche,

30 $\mathrm{Si}$ vedano anche in questo caso i lavori dell'Assemblea Costituente, Commissione per la Costituzione, del 25 ottobre 1946, URL: http://www.camera.it/_dati/costituente/lavori/Commissione/sedoo6/sedoo6.pdf >, pp. 4748. 
che ponesse i partiti come base dell'organizzazione politico-istituzionale del paese, che prevedesse e garantisse la partecipazione popolare.

\section{Due vie democratiche al socialismo?}

Nella costruzione di un discorso pubblico sui temi della nazione e del popolo rintracciamo molte similitudini tra i due partiti, che usano spesso temi, approcci e termini analoghi. Ambedue inscrissero la loro storia nella storia nazionale, il PCF in quella della Rivoluzione del 1789 e della Comune del 1871, il PCI nel Risorgimento. Nei comizi dell'immediato dopoguerra sia in Italia sia in Francia fu posta alla base la necessità di concepirsi come una forza politica nazionale che doveva interessarsi alla nazione e pensare la propria azione politica prima di tutto nel quadro nazionale. Questo si tradusse in primo luogo nella richiesta ai lavoratori di produrre di più nell'interesse della nazione, di mettere momentaneamente da parte le loro rivendicazioni e pensare che la loro lotta passasse attraverso la lotta della rinascita nazionale. Allo stesso tempo però la comparazione mette in luce alcune differenze che mi sembrano sostanziali, e che anche Lazar precisa in Maisons rouges ${ }^{31}$, considerandole però differenze «minimes à l'époque mais lourdes d'enjeux, qui pèseront sur les trajectoires des deux partis»32. Lazar sottolinea che i concetti elaborati nel dopoguerra di "democrazia progressiva" e "democrazia popolare" si distinguono soprattutto per la differente elaborazione da parte dei due segretari: mentre Togliatti avrebbe fatto una distinzione tra "democrazia progressiva" e "dittatura del proletariato", Thorez invece avrebbe fatto delle proposte «frammentarie e poco approfondite» sulla “democrazia nuova e popolare", non distinguendola sostanzialmente dalla dittatura del proletariato. A me sembra che sia necessario mettere in relazione questi concetti con la diversa idea di partito. Mentre l'idea del PCI di "democrazia progressiva" fu sviluppata e sostenuta dal ripensamento del partito in un'ottica di apertura verso le classi medie, e dal dialogo costante con tutti i partiti definiti "democratici”, quindi dall'utilizzo della forza del partito per tentare di allargare la propria capacità d’influenza nella società e in questo senso avviarsi alla costruzione di una democrazia che guardasse al socialismo come "punto di arrivo", l'elaborazione di una "democrazia popolare" del PCF si sviluppò più nel senso di sfruttare al massimo la forza del partito nel dopoguerra per forzare le maglie della democrazia verso il socialismo. Non tanto un gap di elaborazione del concetto, come

${ }^{31}$ LAZAR, Marc, op. cit., pp. 53-57.

32 LAZAR, Marc, op. cit., p. 53. 
rileva Lazar, quanto una diversa visione della strada da percorrere per raggiungere il socialismo attraverso una via non rivoluzionaria. Differenza che ci fa pensare a una certa autonomia dei due partiti rispetto all'URSS (o perlomeno rispetto "all'interpretazione" e all'attuazione delle sue direttive), autonomia che Lazar al contrario non rintraccia. Oltre ai mezzi, anche gli obiettivi dei due partiti nel dopoguerra furono differenti: mentre il PCI volle stabilire una democrazia solida che abbattesse definitivamente il fascismo e per questo puntò molto sulla Costituzione e sull'unità delle forze democratiche, il PCF fece un uso del tutto strumentale dello Stato, cercando di far passare il più possibile modificazioni che spingessero verso la via del socialismo. 


\section{* L'autore}

Martina Martignoni è dottoranda alla School of Management della University of Leicester. Si è interessata di storia dei movimenti in Italia e attualmente si occupa di studi postcoloniali.

Ha contribuito all'organizzazione dei seminari di autoformazione presso l'Università di Bologna "Global Studies" (2009) e "NuS - Nus Frammentazione delle identità fra rimozione storica e riemersione letteraria” (2011). Fa parte del collettivo Bartleby di Bologna.

URL: < http://studistorici.com/progett/autori/\#Martignoni >

\section{Per citare questo articolo:}

MARTIGNONI, Martina, «Due vie democratiche al socialismo? PCI, PCF e il concetto di nazione tra il $1944 \mathrm{e}$ il 1947», Diacronie. Studi di Storia Contemporanea: Quando la classe operaia andava in paradiso, 13/2/2012, URL:< http://www.studistorici.com/2012/02/13/martignoni_numero_9/ >

Diacronie Studi di Storia Contemporanea $\beta$ www.diacronie.it

Risorsa digitale indipendente a carattere storiografico. Uscita trimestrale. redazione.diacronie@hotmail.it

Comitato di redazione: Marco Abram - Giampaolo Amodei - Jacopo Bassi - Luca Bufarale - Alessandro Cattunar - Alice De Rensis Barbara Galimberti - Deborah Paci - Fausto Pietrancosta - Martina Sanna - Matteo Tomasoni - Luca Zuccolo

Diritti: gli articoli di Diacronie. Studi di Storia Contemporanea sono pubblicati sotto licenza Creative Commons 2.5 Possono essere riprodotti a patto di non modificarne i contenuti e di non usarli per fini commerciali. La citazione di estratti è comunque sempre autorizzata, nei limiti previsti dalla legge. 